\title{
New Developments in Respiratory Medicine: a Primary Immunodeficiency perspective
}

Kher $\mathrm{Ng}^{1}$ and John R Hurst ${ }^{2}$

1: Homerton University Hospital NHS Foundation Trust, London, UK

2: UCL Respiratory, University College London, London, UK

Corresponding Author: Professor Hurst.

j.hurst@ucl.ac.uk

+442074726260 


\section{ABSTRACT}

PURPOSE OF REVIEW: To consider recent developments in respiratory medicine that are of relevance to clinicians caring for adults affected by primary immunodeficiency disorders.

RECENT FINDINGS: We consider impulse oscillometry, new bronchoscopic techinques for sampling, MRI and PET, the concept of the human airway microbiome, and new treatment approaches for bronchiectasis and interstitial lung disease to better understand the future of respiratory care for people with PID.

SUMMARY: New approaches to the diagnosis and management of respiratory manifestations of PID have been driven by better understanding of the lung in health and disease, progress in imaging and sampling modalities, and new therapeutics.

KEYWORDS: Primary Immunodeficiency; Lung; Complications

\section{INTRODUCTION}

This review will consider recent developments in respiratory medicine that are of relevance to clinicians caring for adults affected by primary immunodeficiency disorders (PID). We will first summarise the major respiratory manifestations of PID, before going on to describe new approaches to the diagnosis and management of respiratory disease, based on a deeper understanding of the lung in health and disease. Improvements in respiratory health for those living with PID are also being driven by a better understanding of the specific immunodeficiency disorders, for example in relation to genetic diagnosis.

\section{REVIEW}

There are existing reviews that summarise the pulmonary complications PID [1]. In PID characterised by antibody deficiency (PAD) such as Common Variable Immunodeficiency Disorders (CVID), lung complications can be divided into three groups: acute infections of both the upper and lower respiratory tracts, the development of bronchiectasis as a consequence of recurrent airway infection, and granulomatous-lymphocytic interstitial lung disease (GLILD). The latter is best considered the pulmonary component of a multi-system immuno-inflammatory syndrome seen in a subset of people living with CVID $[2,3]$.

People affected by chronic granulomatous disease (CGD) are also prone to recurrent respiratory infections, including those associated with specific organisms such as Staphylococcus, Nocardia and Aspergillus [4]. It is further recognised that people with CGD may develop granulomatous inflammation in the lung (and thoracic lymph nodes) with subsequent risk of interstitial lung disease [5].

Importantly, understanding the pulmonary manifestations of PID is important to both pulmonologists and to clinical immunologists. PID remain associated with significant diagnostic delay and initial manifestations are often within the lung such that pulmonologists need to be alert to the possibility of PID in people presenting with severe, 
unusual, persistent or recurrent ('SPUR') infections, and those with interstitial lung disease. Conversely, clinical immunologists need to be alert to the development of acute and chronic respiratory complications of PID.

From this background, it will be apparent that the major challenges when considering respiratory manifestations of PID relate to the early detection and effective monitoring and treatment of lung complications. In the sections below we will consider new developments in respiratory physiology and imaging, including new opportunities for diagnostic sampling and how our understanding has been affected by a better understanding of the respiratory microbiome. Finally, we will examine new therapeutic approaches to treat both bronchiectasis and GLILD.

\section{Respiratory Physiology}

Spirometry remains the most commonly used pulmonary function test due to its simplicity and wide availability, and is key to the assessment of patients with respiratory disease $[6,7]$. Spirometry is useful to differentiate obstructive diseases (defined by a reduced ratio of forced expiratory volume in one second to (forced) vital capacity - FEV 1 /(F)VC - from restrictive diseases defined by a reduction in total lung capacity (TLC) below the 5 th percentile of predicted value but a normal $\mathrm{FEV}_{1} / \mathrm{VC}$ ratio [8]. Predicted values are derived from the average expected in a healthy individual of any given age, height, sex and race [9].

Reliable spirometry is effort dependent, which can lead to bias. A novel, non-invasive method called the forced oscillation technique (FOT) only requires tidal breathing [10]. FOT, first described by Dubois et al in 1956, uses sound waves of different frequencies superimposed on a patient's tidal breathing to measure the mechanical properties of the airways and lung parenchyma in the form of respiratory impedance $\left(Z_{\mathrm{rs}}\right)$. This technique has similar sensitivity to spirometry in identifying airway abnormalities $[11,12] . Z_{\mathrm{rs}}$ is the sum of all forces, made up of resistance of the airways $\left(R_{\mathrm{rs}}\right)$ and reactance $\left(X_{\mathrm{rs}}\right)$, opposing the generated oscillations $[13,14] . X_{\mathrm{rs}}$ comprises the inertia of the air column (inertance) and the elasticity of the lung (capacitance) and are of opposing phases. Resonant frequency $\left(F_{\text {res }}\right)$ is the frequency at which these two opposing factors are equal $[14,15]$ (Figure 1$)$.

However, FOT measurements were time-consuming as only one frequency wave could be passed at any one time. This led to the development of impulse oscillometry system (IOS) by Michaelson et al in 1975, which sends an impulse comprising multiple sound frequencies into the airways, providing a more accurate mathematical analysis of resistance and reactance $[14,16]$. Simplistically, higher sound frequencies $(20 \mathrm{~Hz})$ only travel shorter distances (thus measuring the larger airways) whilst lower sound frequencies $(5 \mathrm{~Hz})$ travel into the smaller airways. Distal airway obstruction such as that seen in asthma and COPD results in an increase of $R_{\mathrm{rs}}$, and $X_{5}$ (reactance at $5 \mathrm{~Hz}$ frequency) to be more negative, causing the $F_{\text {res }}$ to increase. The change in $R_{\text {rs }}$ is more prominent in asthma and can be used in disease monitoring and assessment of reversibility whereas $X_{5}$ is more impaired in chronic obstructive pulmonary disease (COPD) and can be used to monitor disease progression over time in which it correlates with $\mathrm{FEV}_{1}[15,17]$. 
Similarly, due to decrease in elasticity with subsequent increase in resistance, findings in interstitial lung disease reveal a more negative $X_{5}$ and an increased $R_{\mathrm{rs}}$. It has recently been reported that the inspiratory $X_{5}$ values are more negative than expiratory $X_{5}$ values in interstitial disease, contrasting to the situation in COPD [18].

\section{Diagnostic Sampling}

Enlarged intra-thoracic lymph nodes are seen in many respiratory manifestations of PID, but sampling them has historically been difficult, with significant morbidity associated with mediastinoscopy. In the current practice, thoracic lymphadenopathy can now be more readily defined using endobronchial ultrasound (EBUS) with trans-bronchial needle aspiration (EBUS-TBNA) [19].

There are two types of EBUS currently available: radial probe and linear bronchoscope. Both contain a transducer (which generates and receives ultrasound waves) and a processor (which integrates the sound waves into the ultrasound images) [20]. Radial EBUS was first developed in the 1990s and became commercially available in 1999. Linear EBUS was first developed in 2005 [21]. EBUS is typically performed as a day case procedure using local anaesthesia and conscious sedation.

A radial probe is small and can be inserted through the working channel of a conventional flexible bronchoscope. It has a $360^{\circ}$ rotating transducer which allows visualization of the surrounding layers of the bronchial wall, typically to detect tumour invasion (Figure 2). The probe can also be used to detect peripheral pulmonary lesions. The probe is inserted with a guide sheath and is then left at the site of lesion after removal of the probe to allow insertion of biopsy instrument [21].

The linear EBUS bronchoscope is bulkier and less flexible and has a convex probe at the tip which provides a $60-80^{\circ}$ ultrasound view parallel to the long axis of the scope (Figure 3 ). It can be positioned within the main airways and when in contact with the bronchial wall can assess surrounding lymph nodes and other abnormalities. A 21 or 22-gauge needle can then be introduced into the working channel within the scope, under real-time ultrasound guidance, beyond the bronchial wall into the lymph node or lesions for sampling. [20,21] (Figure 4).

For mediastinal lymph nodes or lesions that are not accessible via the airways, for example those adjacent to the oesophagus, oesophageal ultrasound-guided fine needle aspiration (EUS-FNA) using gastro-intestinal endoscopes can be used [22]. When performed with an EBUS probe, the procedure is called EUS-B [23].

In addition to the ultrasound techniques described above, a further imaging modality that can be used in the assessment of the pulmonary complications of PID is 2-[(18)F]-fluoro-2deoxy-d-glucose positron emission tomography and computed tomography (FDG PET-CT) scanning. PET-CT is now widely used in assessment of malignancy. However, FDG uptake occurs in other conditions with high glucose metabolism such as those associated with inflammation and infection [24]. Although not firmly established as diagnostic modality in PID, PET-CT may have a role in assessing the amount and volume of functional disease 
activity, even in normal-sized lymph nodes (this functional information may therefore be more useful than $\mathrm{CT}$ ), and to inform therapeutic response with repeat PET-CT for comparison [25] (Figure 5). Lesions and mediastinal lymph nodes that demonstrated increased FDG uptake can also be a helpful guide for EBUS sampling of highly active areas, for example where the differential diagnosis includes lymphoma.

There is also emerging evidence on new roles of MRI in the assessment of lung disease in association with PID, mainly due to the advantage of being radiation-free. This is important due to the frequency of monitoring required in PID-related lung diseases. HRCT is currently considered the gold standard for diagnosing and monitoring interstitial lung diseases [26], with the risk of considerable cumulative doses of radiation from repeated CT imaging over a patient's lifetime. There is some evidence that MRI can be considered as an alternative as it is comparable with $\mathrm{CT}$ in the assessment of consolidation, bullae, bronchiectasis, bronchial wall thickening, mucous plugs, nodules and cavities [27]. When used with diffusion weighted imaging (MRI with DWI, Figure 6), this modality has the ability to detect areas of active inflammation even before macroscopic changes become visible on conventional imaging [28]. MRI therefore represents an exciting development in the assessment and monitoring of lung complications in PID. However, advances are limited by the current low availability of MRI scanners, higher cost with low spatial resolution, long scanning time requiring patients' co-operation and the low accessibility to radiologists specializing in chest MRI $[27,29]$.

\section{New Understanding on the Airway Microbiome and Lower Airway Infection:}

Until around 2010, and based on results from culture-dependent microbiology, it was thought that the lower airway was sterile in health. Culture-independent microbiology (16S RNA sequencing) enabled the first report, in 2010 [30], describing a healthy human airway microbiome, dominated by species that had not been grown, sought or reported using lower airway culture. There is now an extensive literature on the airway microbiome in health and disease [31] with, in general, chronic respiratory disease associate with a reduction in the diversity of airway flora, particularly an increase in the prevalence of Proteobacteriaceae - a group comprising species traditionally considered to be respiratory pathogens such as non-typeable Haemophilus influenzae. For people living with chronic respiratory disease such as bronchiectasis, acute infections or 'exacerbations' of those conditions are associated with further dysbiosis [32] again typically characterised by a reduction in the diversity of airway flora.

Currently, most clinical services remain based on culture-dependent methods, increasing supplemented by molecular techniques. Results need to be interpreted in the light of our new understanding of the airway microbiome. Thus, for example, the absence of bacterial growth does not completely exclude bacterial infection, and the presence of a potential pathogen does not necessarily imply that the organism is driving disease (many patients with chronic respiratory disease have persistent organisms in sputum, including Haemophilus. The latter consideration will become more important in relation to culture independent methods. 
A major research question that remains poorly addressed is the nature of the airway microbiome in people with PID, both before and after intervention such as antibody replacement. Whilst there is an evolving literature on the presence and implications of dysbiosis in the gut microbiome in PID [33], there is much less known about the lung [34].

Although recognised as important pathogens in asthma and chronic obstructive pulmonary disease (COPD) for many years, it is now apparent that respiratory viruses can be isolated in more than $50 \%$ of exacerbations in people with CVID are also likely a significant cause of these events [35]. The single commonest virus was human rhinovirus, for which there is currently no effective treatment (or vaccination for those able to make a vaccine response).

Whichever approach to diagnosing respiratory infection is taken, there remains evidence that people with PID delay commencing antibiotics for respiratory infection [35], risking delayed recover, increased severity and the development of complications such as bronchiectasis.

\section{Emerging Therapeutics:}

A significant recent development in respiratory medicine has been the demonstration of efficacy from two anti-fibrotic drugs, pirfenidone [36] and nintedanib [37] in, initially, Usual Interstitial Pneumonia (UIP) but subsequently more widely including nintedanib in those with undifferentiated ILD [38]. Whether such approaches are effective in the context of GLILD remains to be defined.

Revised guidelines for the management of bronchiectasis were published by the European Respiratory Society in 2017 [39]. One of the challenges in interpreting novel research in bronchiectasis is that many studies specifically exclude patients with PID-associated bronchiectasis.

There is increasing evidence to support, and on-going trials to investigate the use of mucoactive therapies in bronchiectasis, as an adjunct to effective airway-clearance physiotherapy, including both nebulised hypertonic saline and carbocisteine [40]. Drugs targeting the cystic fibrosis trans-membrane regulator (CFTR) are transforming the management of $\mathrm{CF}$, and now being explored in non-CF bronchiectasis. Whilst antibiotic prophylaxis, especially with macrolides, is well established in bronchiectasis, novel ways of delivering this include directly to the airway via inhalation which may have benefit in specific patient groups, still to be defined [41]. Bronchiectasis is associated with airway inflammation, and a further novel approach has been to examine novel anti-inflammatories in bronchiectasis, including neutrophil elastase inhibitors and anti-cytokine monoclonal antibodies [42]. Whilst many of these trials have yet to provide outcomes to change the routine management of bronchiectasis, in part because of the heterogeneity inherent in the condition, new interest in bronchiectasis research - in large part driven by the European EMBARC network [43] - is to be broadly welcomed. 
Given that many respiratory infections are caused by respiratory viruses, notably human rhinovirus, the absence of drugs to target rhinovirus infection remains a significant unmet need.

\section{CONCLUSION}

We have considered new approaches to the diagnosis and management of respiratory manifestations of PID, the latter driven by better understanding of the lung in health and disease, particularly the concept of the airway microbiome. These advances are welcome, as are improvements in health through a deeper understanding of the underlying immunodeficiency disorders permitting personalised therapies, but the core message remains that pulmonologists must be alert to the possibility of underlying PID in people presenting with 'SPUR' infections and manifestations of breakdown in immune-regulations, whilst immunologists must be alert to the development of acute and chronic respiratory complications of PID and remain up-to-date in developments in respiratory medicine. Ultimately, multi-professional working across respiratory medicine and immunology is likely to be to the benefit of patient care.

\section{KEY POINTS}

- Immunologists and Pulmonologists should be alert to acute and chronic presentations and complications of respiratory disease in PID

- Better sampling of the lung is available through imaging and endoscopic ultrasound procedures.

- There are new treatments becoming available in bronchiectasis and interstitial lung diseases that have relevance to long -term respiratory complications of PID.

\section{Acknowledgements}

Funding: none.

Conflict of interest: Professor Hurst has received support to attend meetings, and payment both to his employer and personally, for education and advisory work, from pharmaceutical companies that make medicines to treat respiratory disease. 


\section{REFERENCES}

1. Verma N, Grimbacher B, Hurst JR. Lung disease in primary antibody deficiency. Lancet Respir Med. 2015;3(8):651-660. doi:10.1016/S2213-2600(15)00202-7

2. Hurst JR, Verma N, Lowe D, et al. British Lung Foundation/United Kingdom Primary Immunodeficiency Network Consensus Statement on the Definition, Diagnosis, and Management of Granulomatous-Lymphocytic Interstitial Lung Disease in Common Variable Immunodeficiency Disorders. J Allergy Clin Immunol Pract. 2017;5(4):938-945. doi:10.1016/j.jaip.2017.01.021

*3. Hurst JR, Warnatz K; ERS eGLILDnet Clinical Research Collaboration. Interstitial lung disease in primary immunodeficiency: towards a brighter future. Eur Respir J. 2020;55(4):2000089. Published 2020 Apr 3. doi:10.1183/13993003.00089-2020

-new European collaboration on GLILD.

4. Mahdaviani SA, Mohajerani SA, Rezaei N, Casanova JL, Mansouri SD, Velayati AA. Pulmonary manifestations of chronic granulomatous disease. Expert Rev Clin Immunol. 2013;9(2):153-160. doi:10.1586/eci.12.98

5. Kawai $\mathrm{T}$, Watanabe $\mathrm{N}$, Yokoyama $\mathrm{M}$, et al. Interstitial lung disease with multiple microgranulomas in chronic granulomatous disease. J Clin Immunol. 2014;34(8):933-940. doi:10.1007/s10875-0140089-1

*6. Lopes AJ. Advances in spirometry testing for lung function analysis. J Expert Rev Respir Med. 2019;13(6):559-569. doi:10.1080/17476348.2019.1607301

-recent review on Spirometry.

7. Gibson GJ. Spirometry: then and now. Breathe. 2005;1(3):207-216. doi:10.1183/18106838.0103.206

8. Pellegrino $R_{L}$ Viegi G, Brusasco V, et al. Interpretative strategies for lung function tests. Eur Respir J. 2005;26(5):948-968. doi:10.1183/09031936.05.00035205

9. Stanojevic $S$, Quanjer $P$, Miller MR, Stocks J. The Global Lung Function Initiative: dispelling some myths of lung function test interpretation. Breathe. 2013;9(6):462-

474. doi:10.1183/20734735.012113

10. Bickel S, Popler J, Lesnick B, Eid N. Impulse Oscillometry. Chest. 2014;146(3):841847. doi:10.1378/chest.13-1875.

11. Oostveen $\mathrm{E}, \mathrm{MacLeod} \mathrm{D}$, Lorino $\mathrm{H}$, et al. The forced oscillation technique in clinical practice: methodology, recommendations and future developments. Eur Respir J. 2003;22(6):10261041. doi:10.1183/09031936.03.00089403

12. Frantz S, Nihlén U, Dencker M, Engström G, Löfdahl CG, Wollmer P. Impulse oscillometry may be of value in detecting early manifestations of COPD. Respir Med. 2012;106:1116-23. doi:

10.1016/j.rmed.2012.04.010. 
13. Dubois $A B$, Botelho SY, Comroe JH Jr. A new method for measuring airway resistance in man using a body plethysmograph: Values in normal subjects and in patients with respiratory disease. J Clin Invest. 1956;35:327-335. doi: 10.1172/JCl103282

14. Brashier B, Salvi S. Measuring lung function using sound waves: role of the forced oscillation technique and impulse oscillometry system. Breathe. 2015;11(1):57-

65. doi:10.1183/20734735.020514

15. Desiraju K, Agrawal A. Impulse oscillometry: The state-of-art for lung function testing. Lung India. 2016;33:410-416. doi: 10.4103/0970-2113.184875

16. Michaelson ED, Grassman ED, Peters WR. Pulmonary mechanics by spectral analysis of forced random noise. J Clin Invest. 1975;56:1210-1230. doi: 10.1172/JCI108198

17. Gong SG, Yang WL, Zheng W, Liu JM. Evaluation of respiratory impedance in patients with chronic obstructive pulmonary disease by an impulse oscillation system. Mol Med Rep.

2014;10:2694-2700. doi: 10.3892/mmr.2014.2528

18. Takeichi N, Yamazaki H, Fujimoto K. Comparison of impedance measured by the forced oscillation technique and pulmonary functions, including static lung compliance, in obstructive and interstitial lung disease. Int J Chron Obstruct Pulmon Dis. 2019;14:1109-1118. Published 2019 May 24. doi:10.2147/COPD.S198030

19. Wahidi MM, Herth F, Yasufuku K, et al. Technical aspects of endobronchial ultrasound-guided transbronchial needle aspiration: CHEST guideline and expert panel report. Chest. 2016;149(3):816835. doi:10.1378/chest.15-1216

20. Balamugesh T, Herth FJ. Endobronchial ultrasound: A new innovation in bronchoscopy. Lung India. 2009:26(1);17-21. doi: 10.4103/0970-2113.45199

21. Gomez M, Silvestri GA. Endobronchial Ultrasound for the Diagnosis and Staging of Lung Cancer. Proceedings of the American Thoracic Society. 2009: 6(2), 180-186. doi:10.1513/pats.200808-081lc

22. Detterbeck FC, Lewis SZ, Diekemper R, et al. Executive Summary: Diagnosis and management of lung cancer, 3rd ed: American College of Chest Physicians evidence-based clinical practice guidelines. Chest. 2013;143:7S-37S. doi:10.1378/chest.12-2377

23. Colella S, Scarlata S, Bonifazi M, et al. Biopsy needles for mediastinal lymph nodes sampling by endosonography: current knowledge and future perspectives. J Thorac Dis. 2018;10(12):6960-6968. doi: $10.21037 /$ jtd.2018.11.35

*24. Rahman WT, Wale DJ, Viglianti BL, et al. The impact of infection and inflammation in oncologic 18F-FDG PET/CT imaging. Biomed Pharmacother. 2019;117; 109168.

doi:10.1016/j.biopha.2019.109168

-recent review on PET-CT.

25. Jolles $S$, Carne $E$, Brouns $M$, et al. FDG PET-CT imaging of therapeutic response in granulomatous lymphocytic interstitial lung disease (GLILD) in common variable immunodeficiency (CVID). Clin Exp Immunol. 2016;187(1):138-145. doi:10.1111/cei.12856

26. Cinetto F, Scarpa R, Rattazzi M, et al. The broad spectrum of lung diseases in primary antibody deficiencies. Eur Respir Rev 2018;27(149):180019. doi:10.1183/16000617.0019-2018 
27. Arslan S, Poyraz N, Ucar R, et al. Magnetic Resonance Imaging May Be a Valuable Radiation-Free Technique for Lung Pathologies in Patients with Primary Immunodeficiency. J Clin Immunol. 2015;36(1):66-72. doi:10.1007/s10875-015-0227-4

28. Milito C, Pulvirenti F, Serra G, et al. Lung Magnetic Resonance Imaging with Diffusion Weighted Imaging Provides Regional Structural as well as Functional Information Without Radiation Exposure in Primary Antibody Deficiencies. J Clin Immunol. 2015; 35:491-500. doi:10.1007/s10875-015-0172-2

*29. Baumann U, Routes JM, Soler-Palacín P, et al. The Lung in Primary Immunodeficiencies: New Concepts in Infection and Inflammation. Front Immunol. 2018;9:1837.

doi:10.3389/fimmu.2018.01837

-recent review on PID and lung.

30. Hilty $\mathrm{M}$, Burke $\mathrm{C}$, Pedro $\mathrm{H}$, et al. Disordered microbial communities in asthmatic airways. PLoS One. 2010;5(1):e8578. Published 2010 Jan 5. doi:10.1371/journal.pone.0008578

31. The Lung Microbiome. Edited by Michael J. Cox, Markus J. Ege and Erika von Mutius. 2019. DOI: 10.1183/2312508X.erm8319. ISBN (electronic): 978-1-84984-102-3

32. Cox MJ, Turek EM, Hennessy C, et al. Longitudinal assessment of sputum microbiome by sequencing of the $16 \mathrm{~S}$ rRNA gene in non-cystic fibrosis bronchiectasis patients. PLoS One. 2017;12(2):e0170622. Published 2017 Feb 7. doi:10.1371/journal.pone.0170622

33. Berbers RM, Nierkens S, van Laar JM, Bogaert D, Leavis HL. Microbial Dysbiosis in Common Variable Immune Deficiencies: Evidence, Causes, and Consequences. Trends Immunol. 2017;38(3):206-216. doi:10.1016/j.it.2016.11.008

34. Mooney D, Edgar D, Einarsson G, Downey D, Elborn S, Tunney M. Chronic lung disease in common variable immune deficiency (CVID): A pathophysiological role for microbial and non-B cell immune factors. Crit Rev Microbiol. 2017;43(4):508-519. doi:10.1080/1040841X.2016.1268568

35. Sperlich JM, Grimbacher B, Workman S, et al. Respiratory Infections and Antibiotic Usage in Common Variable Immunodeficiency. J Allergy Clin Immunol Pract. 2018;6(1):159-168.e3. doi:10.1016/j.jaip.2017.05.024

36. King TE Jr, Bradford WZ, Castro-Bernardini S, et al. A phase 3 trial of pirfenidone in patients with idiopathic pulmonary fibrosis [published correction appears in N Engl J Med. 2014 Sep 18;371(12):1172]. N Engl J Med. 2014;370(22):2083-2092. doi:10.1056/NEJMoa1402582

37. Richeldi L, du Bois RM, Raghu G, et al. Efficacy and safety of nintedanib in idiopathic pulmonary fibrosis [published correction appears in N Engl J Med. 2015 Aug 20;373(8):782]. N Engl J Med. 2014;370(22):2071-2082. doi:10.1056/NEJMoa1402584

*38. Flaherty KR, Wells AU, Cottin V, et al. Nintedanib in Progressive Fibrosing Interstitial Lung Diseases. N Engl J Med. 2019;381(18):1718-1727. doi:10.1056/NEJMoa1908681

- use of anti-fibrotics in other interstitial lung diseases.

39. Polverino E, Goeminne PC, McDonnell MJ, et al. European Respiratory Society guidelines for the management of adult bronchiectasis. Eur Respir J. 2017;50(3):1700629. Published 2017 Sep 9. doi:10.1183/13993003.00629-2017 
*40. Bradley JM, Anand R, O'Neill B, et al. A $2 \times 2$ factorial, randomised, open-label trial to determine the clinical and cost-effectiveness of hypertonic saline (HTS 6\%) and carbocisteine for airway clearance versus usual care over 52 weeks in adults with bronchiectasis: a protocol for the CLEAR clinical trial. Trials. 2019;20(1):747. Published 2019 Dec 19. doi:10.1186/s13063-019-3766-9

-new landmark trial protocol in bronchiectasis.

41. Haworth CS, Bilton D, Chalmers JD, et al. Inhaled liposomal ciprofloxacin in patients with noncystic fibrosis bronchiectasis and chronic lung infection with Pseudomonas aeruginosa (ORBIT-3 and ORBIT-4): two phase 3, randomised controlled trials [published correction appears in Lancet Respir Med. 2019 Jan 25;:]. Lancet Respir Med. 2019;7(3):213-226. doi:10.1016/S2213-2600(18)30427-2

*42. Keir HR, Fong CJ, Crichton ML, et al. Personalised anti-inflammatory therapy for bronchiectasis and cystic fibrosis: selecting patients for controlled trials of neutrophil elastase inhibition. ERJ Open Res. 2019;5(1):00252-2018. Published 2019 Mar 25. doi:10.1183/23120541.00252-2018

- novel anti-inflammatory drugs for bronchiectasis.

43. Chalmers JD, Crichton M, Goeminne PC, et al. The European Multicentre Bronchiectasis Audit and Research Collaboration (EMBARC): experiences from a successful ERS Clinical Research Collaboration. Breathe (Sheff). 2017;13(3):180-192. doi:10.1183/20734735.005117 


\section{FIGURE LEGENDS}

Figure 1: Schematic illustration of changes to resistance (Rrs) and reactance in airway obstruction. From reference [11], with permission.

Figure 2: (A) A 20-MHz miniature radial probe with the balloon sheath on the tip inflated with water, inserted through a 2.8- $\mathrm{mm}$ working channel of a flexible bronchoscope. (B) Radial probe ultrasonic image. From reference [21], with permission.

Figure 3: A schematic of a convex probe endobronchial ultrasound (EBUS) system. From reference [21], with permission.

Figure 4: (A) Convex probe EBUS in the main airway. (B) Ultrasound image shows needle in lymph node (entering from top right). From reference [21], with permission.

Figure 5: FDG PET-CT pre- and 3 months post-rituximab and mycophenolate treatment. (a) Maximum intensity projection (MIP) whole body images pre-treatment showing widespread abnormal uptake of tracer in the lung parenchyma and lymph nodes above and below the diaphragm. (b) MIP images following treatment show near resolution of all the areas of abnormal tracer uptake. (c,d) Axial fused PET/CT images at the mid-thoracic level. The level of FDG uptake is represented by the intensity of colour superimposed upon the CT image. (c) There is a combination of interstitial septal thickening and ill-defined FDG avid peribronchovascular nodules. (d) Post-treatment images at the same level shows improvement of the nodularity with near resolution of abnormal FDG uptake within them. (e) Images at the level of the carina show an enlarged lymph node exhibiting intense abnormal FDG uptake prior to treatment. (f) Following treatment, the lymph node at this site normalizes in size and shows no abnormal tracer uptake. (g) Pre-treatment images through the abdomen at the level of the right renal hilum demonstrate numerous enlarged FDG avid retroperitoneal lymph nodes. (h) These have reduced in size and FDG uptake following treatment. From reference [25], permission requested.

Figure 6: Representative MRI with DWI sequences: nodules. (a) MRI BLADE sequence detecting a nodule (white arrow) at the right lower lobe. (b) Without a DWI corresponding hotspot. (c) MRI BLADE sequence detecting multiple and bilateral small nodules (white arrow) of the lower lobes and (d) their corresponding hotspots at DWI assessment (white arrow). From reference [28] with permission. 